\section{EMBRYRIDDLE}

Aeronautical University

SCHOLARLY COMMONS
International Journal of Aviation, Aeronautics, and Aerospace

\title{
Remote Pilot Situational Awareness with Augmented Reality Glasses: An Observational Field Study
}

Jeffery Coleman

ERAU, colemj21@my.erau.edu

David Thirtyacre

ERAU, thirtyad@erau.edu

Follow this and additional works at: https://commons.erau.edu/ijaaa

Part of the Aviation Safety and Security Commons, Management and Operations Commons, and the Other Aerospace Engineering Commons

\section{Scholarly Commons Citation}

Coleman, J., \& Thirtyacre, D. (2021). Remote Pilot Situational Awareness with Augmented Reality Glasses: An Observational Field Study. International Journal of Aviation, Aeronautics, and Aerospace, 8(1). https://doi.org/10.15394/ijaaa.2021.1547

This Article is brought to you for free and open access by the Journals at Scholarly Commons. It has been accepted for inclusion in International Journal of Aviation, Aeronautics, and Aerospace by an authorized administrator of Scholarly Commons. For more information, please contact commons@erau.edu. 
Augmented Reality (AR) devices, tools that can overlay elements of a synthetic environment while providing data visualization, are increasing in both recreational popularity and commercial application (Akçayır et al., 2016). Drone operators within the public-safety domain have been employing AR to assist in search and rescue (SAR), and in the oil and gas industry to assist in pipeline inspections. Both examples however, limit the use of AR to sensor management and target area situational awareness (SA) rather than flight control, 14 CFR Part 107 compliance, and ultimately flight safety (Shaw, 2018). Incorporation of AR into the flight control system may enable the sUAS operator to simultaneously observe the aircraft while interfacing with the GCS. This paper evaluates the effectiveness of AR as a means to increase sUAS pilot situational awareness by minimizing heads-down time during various phases of flight.

As a pilot's cognitive load increases and decreases during various phases of flight, AR has the potential to reduce the pilot's requirement to look away from the aircraft to retrieve flight and sensor information (Hitchcock \& Slung, 2018). Changing head orientation and eye focus to the GCS often makes re-acquiring the visual location of the sUAS challenging, subsequently adding to the pilot's cognitive load and reducing SA.

The amount of information sUAS pilots require in the execution of their missions has been steadily increasing as platform and sensor capabilities improve. CFR Part 107 requires that pilots maintain a visual line of sight (VLOS) with the aircraft, which is difficult given that much of the flight data, such as telemetry and sensor information, is presented on a phone, tablet or computer screen (Federal Aviation Administration, 2016). The requirement to look away from the aircraft to ingest flight information is similar to a traditional pilot's instrument crosscheck, which saw the introduction of a HUD to minimize "heads-down" time (Iwaneczko et al., 2016). While numerous studies have explored the potential application of VR and AR devices for elements of sUAS flight, none have explored the effects of such devices on positional situational awareness and maintenance of VLOS. This observational field study analyzed the sUAS pilot's physical cross-check between the GCS display and the aircraft through video recording with and without the use of an AR device shown in Figure 1. 


\section{Figure 1}

Epson Moverio BT-300

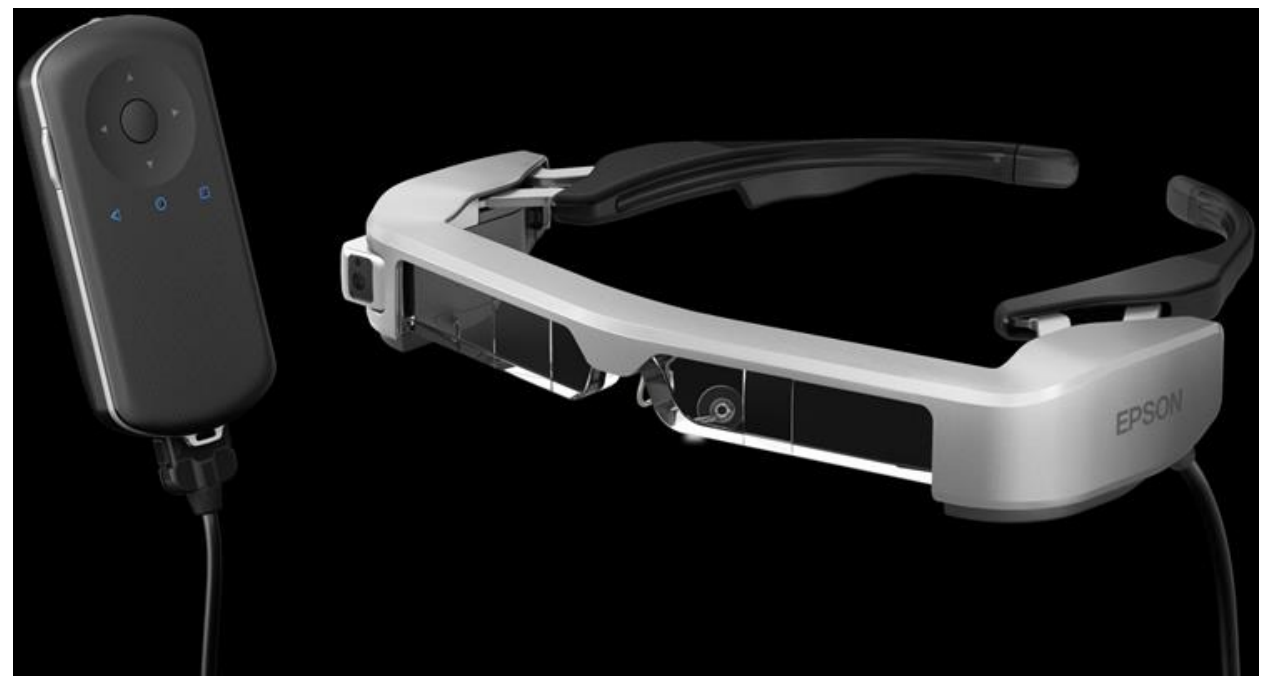

\section{Limitations}

There were several limitations and delimitations of this observational study. Endsley and Jones (2012) defined SA as "the perception of the elements in the environment within a volume of time and space, the comprehension of their meaning, and the projection of their status in the near future" (p. 13).

There are three levels of SA:

- Level 1-Perception of the elements in the environment

- Level 2-Comprehension of the current situation

- Level 3-Projection of the future status

Situational Awareness is a requirement for proper aeronautical decision making and linked directly to performance (Endsley \& Jones, 2012). While defining SA is straight forward, assessing SA can be a complex endeavor. This study only considered the elements in the environment used to define Level $1 \mathrm{SA}$ and whether those elements were available in the pilot's field of view.

Data collection took place during a training event spanning several days. The pilots were all experienced in manned and unmanned flight and flew in both automated and manual modes. In most cases, this was the first time the pilots employed an AR device, which may have influenced their cross-check. The dependent variable was limited to three categories which cannot be directly correlated with situational awareness. While some assumptions can be made as to the ingestion of critical information required for situational awareness based on pilot head position, direct measurements of SA was not attempted. 


\section{Related Research}

For the last decade, researchers have studied the application of VR and $\mathrm{AR}$ in various sUAS flight scenarios. The bulk of the research has shown positive effects to either manual operation of sUAS or increased efficiency in autonomous flight planning. Researchers from the University of California at Berkley tested a VR immersive 3-dimensional (3D) interface on aerial route planning with respect to safety, efficiency, and usability. While they found that using a VR interface provided comparable safety and usability results over manual interfaces, they indicated that a VR interface significantly reduced flight path planning times when compared to a 2-dimensional touchscreen (Paterson et al., 2019). Interestingly, through participant surveys they also found a preference for a VR interface over manual control. However, participants' VR experience did not correlate with performance.

Similar research conducted by a team from Graz University in 2013 revealed that the application of $\mathrm{AR}$ visualization increased operator situational awareness as demonstrated by increased accuracy of position estimates when compared to solely relying on visual observations (Zollmann et al., 2013). While they lacked the number of participants to make definite conclusions, they did speculate that the use of AR positively impacted situational awareness at farther distances.

Researchers at the University of Wisconsin-Stevens Point sought to investigate opportunities and dangers present in the current human interfaces used to operate commercial drones utilizing first person view (FPV) headsets. Notably, participants reported an uneasiness during ascent because they could not determine the proximity to vertical obstructions, and because the FPV headsets obscured their natural vision of the sUAS (Hall et al., 2018). However, despite concerns with FOV and safety, the study found that the use of an FPV device provided the most accurate target sensor images.

\section{Methodology}

This field study employed video-based observational methods to quantitatively determine the ratio of sUAS pilot time-sharing between viewing the aircraft and interaction with the GCS. Approximately five hours of video recordings provided source data, and each video was categorized according to type of control (manual or automated) and AR device (with or without). Statistical T-tests were applied to determine if there was a statistically significant difference between the mean time the aircraft was in the pilot's FOV, with and without an AR device.

\section{Sample}

The sample for this study was drawn from video recordings of automated and manual flights conducted by experienced sUAS pilots from Embry-Riddle 
Aeronautical University. All flights were performed in the same location in October, 2019, as part of other concurrent academic studies. Flights were separated into automated flights with an AR device, automated flights without an AR device, and manual flights with an AR device. There were 16 recordings of automated flights without an AR device $(n=16)$ and 10 recordings of automated flights with an AR device $(n=10)$. Additionally, nine manually controlled flights with an AR device were evaluated. However, since no manually controlled flights were conducted without an AR device, no statistical comparison was performed. Measurements were taken using frame counts and a digital stopwatch to assess observed head and eye position of the pilot. Each recording was scored multiple times to improve reliability.

\section{Apparatus and Materials}

Video for the field study was captured using a stand-alone high-definition camera. Pilots used various sUAS platforms such as DJI's Inspire 1, Inspire 2, Mavic 2, Mavic Pro, Phantom 4, and Parrot's Bebop-2. Flights were programmed and controlled using Pix4D Capture and DJI's Go 4 application on tablets connected to the respective remote controllers. During AR enabled flights, operators used Epson's Moverio BT-300 or BT-35E smart glasses connected via HDMI or USB.

The AR device repeated most of the information on the GCS display including the camera image and telemetry data. In cases where the AR device could display different information (e.g., Inspire-2) the display was set to the main camera. The resolution of the camera displayed in the GCS was set to 1080p for standardization. Various visors of different tints were available and selected by pilot preference. The AR device was worn prior to takeoff until after landing.

\section{Results}

Graphical depictions of the automated flight data are displayed in Figure 2 (without AR) and Figure 3 (with AR). The data in Figure 4 are during manual flight, with AR. In all figures, the percentage of attention focused on the GCS is in red while the time focused on the aircraft is in blue. Distractions, or the time the evaluators could not determine where the pilot's attention was focused, is displayed in yellow. 


\section{Figure 2}

\section{Pilot Attention during Automated Flights without AR Device}

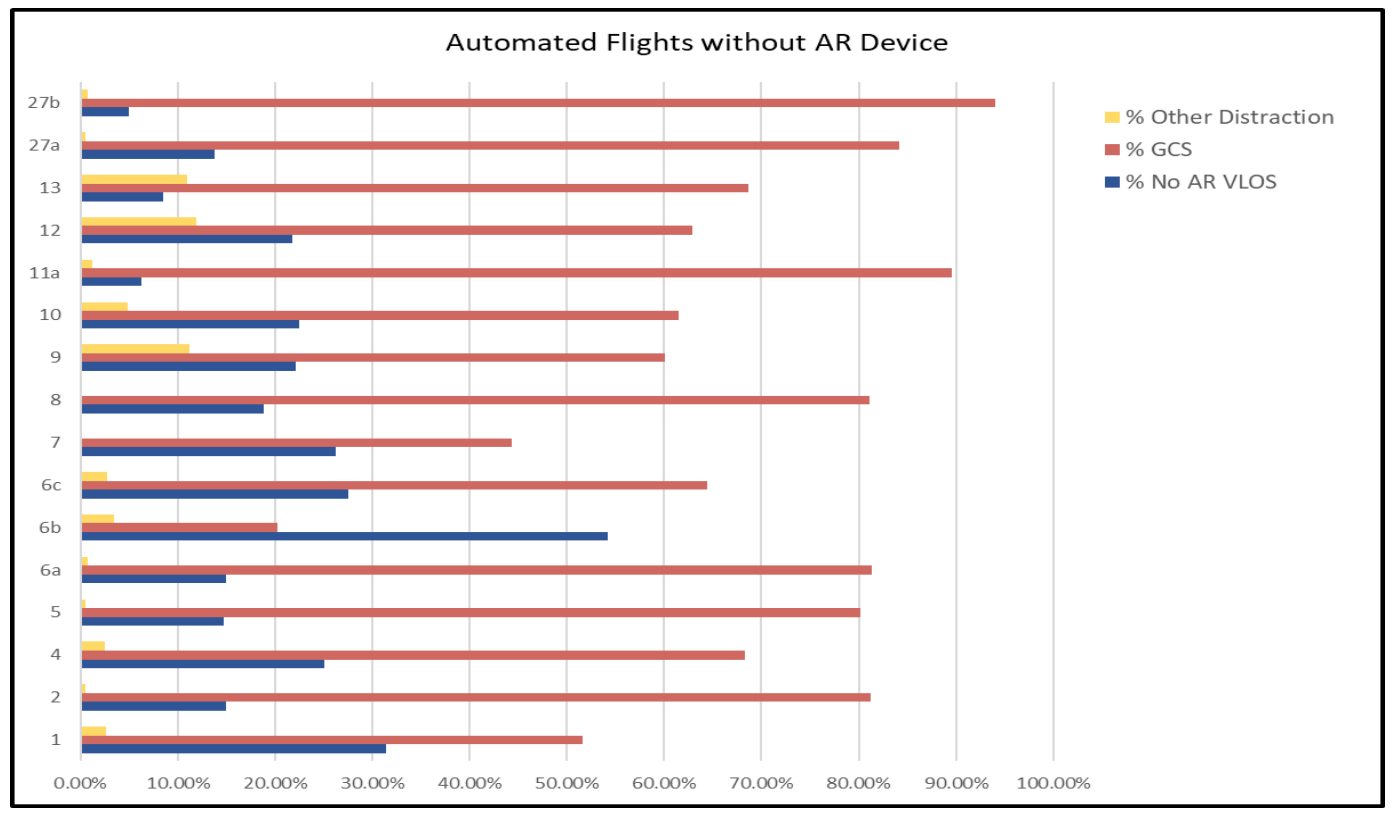

Figure 3

Pilot Attention during Automated Flights with AR Device

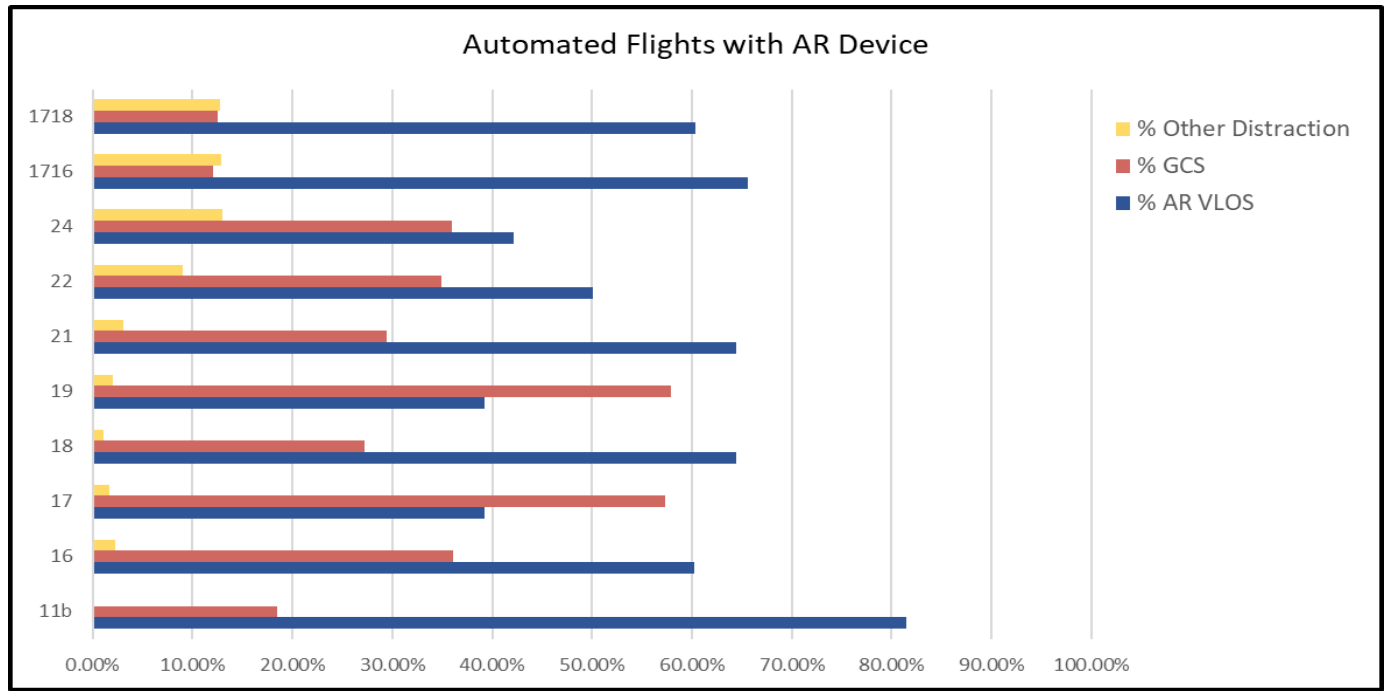


The descriptive statistics for the automated flight data in Figure 2 and Figure 3 illustrate that without AR, the pilots focused on the GCS display $68.4 \%$ of the time, while spending $20.5 \%$ of the time with the aircraft in the pilot's FOV. When AR was included, the pilot's focus changed to $32.2 \%$ on the GCS and $56.7 \%$ on the aircraft. A $T$-Test was preformed to determine if there was a statistically significant difference between flights with or without the AR device.

The t-test indicated a significant difference, $t(9)=6.3, p<.001$ in means between GCS focused time with participants wearing the AR device $(M=32.2$, $S D=16.1)$ compared to the samples without AR $(M=68.4, S D=18.8)$. There was also a significant difference, $t(9)=13.1, p<.001$ in the time focused on the aircraft with $\mathrm{AR}(M=56.7, S D=13.7)$ compared to without $\mathrm{AR} \quad(M=20.5$, $S D=11.8)$.

The data set for manual flight did not include fights without AR (i.e., all the flights were performed with the AR device). The flight profile included maneuvering to several locations and capturing images of different objects then moving to another position and repeating the process. This profile was selected due to the inherent heads-down time spent focused on the GCS display while maneuvering to a precise location (position and altitude) and capturing images. The profile was typical of a flight with the intent of capturing video and images. The results for manual flight are depicted in Figure 4. The descriptive statistics revealed that the pilots spent $96.3 \%$ of their time focused on the aircraft while $2.2 \%$ of their time looking at the GCS.

\section{Figure 4}

\section{Pilot Attention during Manual Flights with AR Device}

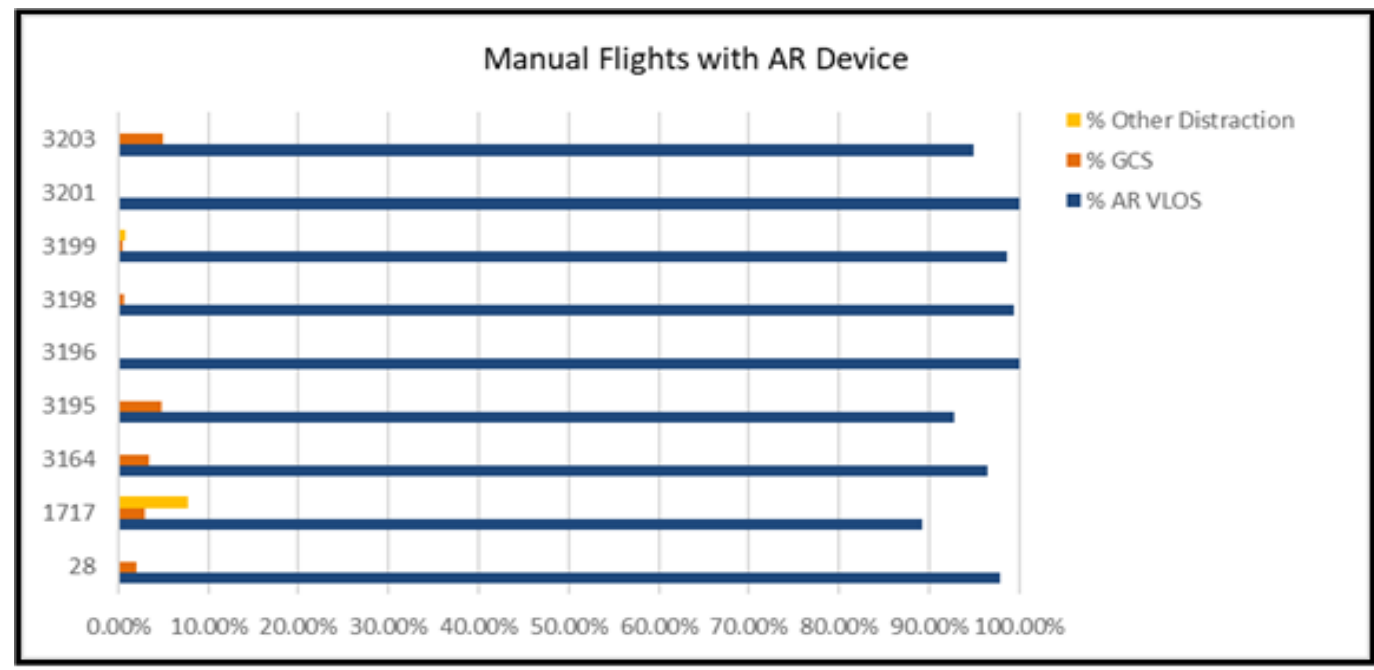




\section{Discussion}

The analysis of the recorded data clearly indicates that conducting sUAS flight operations with the aid of an AR device significantly increased the amount of time pilots were able to keep the aircraft in their FOV. Having access to flight telemetry, aircraft position, and the aircraft camera reduced the amount of headsdown time similarly to the benefits of using a heads-up display (Shelton et al., 2015). Additionally, the typical aircraft-to-GCS crosscheck to read parameters such as altitude and battery remaining, were minimized and continually available through AR. This allowed the pilots to keep the aircraft in their FOV while interpreting the display information. This information is critical for all levels of SA.

Although there were outliers, in all cases, the AR device reduced the amount of time the aircraft was outside of the pilots FOV when compared to nonAR flights. The outliers were attributed to external environmental factors and operator preference. As pilots became accustomed to the flight data presented on the AR device, their individual controlling techniques appeared to change to maximize both comfort and efficiency in managing their cross-check. During the course of the observational study, the participants comfort levels anecdotally appeared to improve with experience. For example, device cable management (from the AR device and the glasses) improved, dual use of prescription glasses became easier, and operators implemented personal system configurations such as the use of tinted lenses to counter environmental brightness.

The introduction of the AR devices nearly reversed the time-sharing ratio between the aircraft and GCS interaction while flying automated routes. Participants were noted to have commented on the increased ease of monitoring the pre-programmed patterns while wearing the devices. However, an increased work load to configure and program the AR device (e.g., cable management, AR configuration, and battery levels) was noted over the typical GCS tablet configuration. In some cases, there was noticeable pilot frustration when attempting to bring the AR system online.

The results for the manual flights were surprising. The image capture process is typically preformed heads-down, but the results indicate that the AR display was of sufficient resolution and brightness for the pilots to capture all of the images without referring to the GCS display. This may have been due to the typical reflection on the GCS tablet in bright sun making it difficult to interpret. Analyzing the voice recordings and comments from the pilots confirmed that they felt it was easier to see the AR display versus the GCS tablet display.

The introduction of AR devices during sUAS flight has demonstrated similar capabilities to the inclusion of the HUD in military and commercial aircraft. It can be anticipated that their inclusion in routine sUAS operations will continue to increase as the hardware becomes less expensive, more capable, 
accessible, and integrated with other control software interfaces. The economic benefits of such AR devices will most certainly provide a return on investment as they are introduced to drone operations in industries such as agriculture, survey, and construction. Lastly, with the development of swarm technology, it is believed that AR interfaces have the potential to reduce pilot cognitive load enough to enable the operator to control multiple sUAS devices simultaneously (Li et al., 2015).

\section{Conclusion}

The observational evidence of the benefits of AR devices highlights the potential of such devices to reduce the cognitive load of pilots by reducing the amount of time focused on the GCS display and away from the aircraft. The introduction of AR not only reduced both the amount of time spent visually interacting with the GCS, but also increased the amount of time pilots were able to maintain the aircraft in their FOV.

The results of this field study provided insight into the effectiveness of AR devices in sUAS operations. While observing pilots in an unobtrusive natural environment was beneficial, there remains a requirement to conduct further studies under a true experimental design. A controlled environment in which sUAS pilots are given explicit tasks and parameters, conducted under a more rigid set of conditions (including consistent AR devices, sUAS platforms, and control software) would provide greater internal validity. 


\section{References}

Akçayır, M., Akçayır, G., Pektaş, H. M., \& Ocak, M. A. (2016). Augmented reality in science laboratories: The effects of augmented reality on university students' laboratory skills and attitudes toward science laboratories. Computers in Human Behavior, 57, 334-342. doi:10.1016/j.chb.2015.12.054

Endsley, M., \& Jones, D. (2012). Designing for situational awareness: An approach to user-centered design (2nd ed.). CRC.

Federal Aviation Administration. (2016). Summary of small unmanned aircraft rule (Part 107). FAA News, June 21st, 2016. Retrieved from https://www.faa.gov/uas/media/Part_107_Summary.pdf.

Hall, B., Anderson, N., \& Leaf, K. (2017). Improving human interfaces for commercial camera drone systems. Proceedings of the $2017 \mathrm{CHI}$ Conference Extended Abstracts on Human Factors in Computing Systems, May 06-11, Denver, CO, USA. doi:10.1145/3027063.3048428

Hitchcock, A., \& Slung, K. (2018). Multi-view augmented reality with a drone. Proceedings of ACM Symposium on Virtual Reality Software and Technology (VRST). ACM, Tokyo, Japan, November 28th, 2018. doi.org/10.1145/3281505.3283397

Iwaneczko, P., Jedrasiak, K., \& Nawrat, A. (2016). Augmented reality in UAVs applications. Innovative Simulation Systems, Studies in Systems, Decision and Control. 33, 2016. doi:10.1007/978-3-319-21118-3_6

Li, N., Cartwright, S., Nittala, A., Sharlin, E., \& Sousa, M. (2015). Flying frustum: A spatial interface for enhancing human-UAV awareness. Proceedings of the 3rd International Conference on Human-Agent Interaction, Daegu, Kyungpook, Republic of Korea, October 21-24, 2015. doi:10.1145/2814940.2814956

Paterson, J., Han, J., Cheng, T., Laker, P., McPherson, D., Menke, J., \& Yang, A. (2019). Improving usability, efficiency, and safety of UAV path planning through a virtual reality interface. arXiv:1904.08593, April 18th, 2019. Retrieved from https://arxiv.org/pdf/1904.08593.pdf

Shaw, K. (2018). Augmented reality drone overlays feed data to first responders. Robotics Business Review, 2018. Retrieved from https://www.roboticsbusinessreview.com/unmanned/augmented-realitydrone-overlays-feed-data-to-first-responders.

Shelton, K., Arthur III, J., Prinzel III, L., Nicholas, S., Williams, S., \& Bailey, R. (2015). Flight test of a head-worn display as an equivalent-HUD for terminal operations. SPIE Proceedings, 9470, Display Technologies and Applications for Defense, Security, and Avionics IX; and Head- and Helmet-Mounted Displays XX, 21 May 2015. doi:10.1117/12.2177059

Zollmann, S., Hoppe, C., Langlotz, \& Reitmayr, G. (2013). FlyAR: Augmented 
reality supported micro aerial vehicle navigation. IEEE Transactions on Visualization and Computer Graphics, 20(4), 560-568.

doi:10.1109/TVCG.2014.24 